\title{
Statistical evaluation of the flux cross-calibration of the XMM-Newton EPIC cameras
}

\author{
S. Mateos ${ }^{1}$, R. D. Saxton ${ }^{2}$, A. M. Read ${ }^{1}$, and S. Sembay ${ }^{1}$ \\ 1 Department of Physics and Astronomy, University of Leicester, University Road, Leicester, UK \\ e-mail: sm279@star.le.ac.uk \\ 2 XMM SOC, ESAC, Apartado 78, 28691 Villanueva de la Cañada, Madrid, Spain
}

Received 24 November 2008 / Accepted 20 January 2009

\begin{abstract}
Context. The second XMM-Newton serendipitous source catalogue, 2XMM, provides the ideal data base for performing a statistical evaluation of the flux cross-calibration of the XMM-Newton European Photon Imaging Cameras (EPIC).

Aims. We aim to evaluate the status of the relative flux calibration of the EPIC cameras on board the XMM-Newton observatory (MOS1, MOS2, and pn) and investigate the dependence of the calibration on energy (from 0.2 to $12.0 \mathrm{keV}$ ), position of the sources in the field of view of the X-ray detectors, and lifetime of the mission.

Methods. We compiled the distribution of flux percentage differences for large samples of "good quality" objects detected with at least two of the EPIC cameras. The mean offset of the fluxes and dispersion of the distributions was then found by Gaussian fitting. Count rate to flux conversion was performed with a fixed spectral model. The impact on the results of varying this model was investigated. Results. Excellent agreement was found between the two EPIC MOS cameras to better than $4 \%$ over the entire energy range where the EPIC cameras are best calibrated (0.2-12.0 keV). We found that MOS cameras register 7-9\% higher flux than EPIC pn below $4.5 \mathrm{keV}$ and a $10-13 \%$ flux excess at the highest energies $(\gtrsim 4.5 \mathrm{keV})$. No evolution of the flux ratios is seen with time, except at the lowest energies $(\lessgtr 0.5 \mathrm{keV})$, where we found a strong decrease in the MOS to pn flux ratio with time. This effect is known to be due to a gradually degrading MOS redistribution function. The flux ratios show some dependence on distance from the optical axis in the sense that the MOS to pn flux excess increases with off-axis angle. Furthermore, in the 4.5-12.0 keV band there is a strong dependence of the MOS to pn excess flux on the azimuthal-angle. These results strongly suggest that the calibration of the Reflection Grating Array (RGA) blocking factors is incorrect at high energies. Finally, we recommend ways to improve the calculation of fluxes in future versions of XMM-Newton serendipitous source catalogues.
\end{abstract}

Key words. instrumentation: detectors - methods: statistical - space vehicles: instruments - X-rays: general

\section{Introduction}

ESA's XMM-Newton observatory (Jansen et al. 2001) was launched in December 1999. XMM-Newton carries on board three identical Wolter type 1 telescopes with 58 nested mirror shells. At the focal plane of each mirror there is a European Photon Imaging Camera (EPIC) that records images and spectra of celestial X-ray sources (Strüder et al. 2001; Turner et al. 2001). Two of the cameras use front-illuminated EPIC-MOS (Metal-Oxide Semi-conductor) CCDs as X-ray detectors (hereafter, MOS1 and MOS2), while the third camera uses an EPIC-pn (p-n-junction) CCD (hereafter, pn). EPIC has a fieldof-view (FOV) of 30' diameter, moderate energy resolution and can be operated in various observational modes related to the readouts in each mode (Kendziorra et al. 1997, 1999; Kuster et al. 1999; Ehle et al. 2001). The two telescopes with the MOS cameras at the focal plane have a Reflection Grating Spectrometer mounted behind the mirrors (RGS, den Herder et al. 2001). The incident light is split roughly 50\%:50\% between the RGS and MOS cameras.

The absolute and relative calibration of the XMM-Newton instruments has been monitored and studied since launch. These studies are mostly based on detailed spectral analyses of bright "calibration" targets that have been observed regularly by XMM-Newton. The relative flux calibration of the EPIC cameras is known to be better than 7\% on-axis, with MOS fluxes being higher than pn fluxes (see Stuhlinger et al. 2008), and has been shown to have both time and energy dependence. For details on the calibration of other XMM-Newton science instruments see Stuhlinger et al. (2008).

In this work we use a large sample of serendipitous X-ray sources to study the flux cross-calibration of the EPIC cameras as a function of various parameters. We investigated the performance of the EPIC instruments by carrying out a systematic analysis of the camera-to-camera flux ratio for a large number of sources with "good quality" X-ray data in various energy bands over the XMM-Newton lifetime. We compare the fluxes measured by the EPIC cameras in different energy bands covering the entire energy range from $0.2 \mathrm{keV}$ to $12.0 \mathrm{keV}$, for sources detected at different epochs and detected at different positions in the field of view. In this way we can investigate how instrumental effects such as the detector quantum efficiency, point spread function (PSF) and mirror vignetting function affect the relative flux calibration of the EPIC cameras. This is an important complement to studies based upon bright targets, which are necessarily restricted to the central CCD of the MOS cameras. The 
outer MOS CCDs are operated exclusively in FullFrame mode (i.e. PrimeFullWindow mode) which has a pile-up threshold of $0.7 \mathrm{cts} / \mathrm{s}^{1}$.

For this analysis we have used the sources in the second XMM-Newton serendipitous source catalogue, 2XMM (Watson et al. 2008). 2XMM is the largest catalogue of X-ray sources compiled to date, containing more than 240000 sources, and therefore provides the ideal data base to conduct this study. Furthermore, we have used the same energy band definition as in 2 XMM: $0.2-0.5 \mathrm{keV}, 0.5-1.0 \mathrm{keV}, 1.0-2.0 \mathrm{keV}, 2.0-4.5 \mathrm{keV}$ and $4.5-12.0 \mathrm{keV}$.

This paper is organised as follows: in Sect. 2 we give a short description of the source detection procedure used to compile the 2XMM catalogue (Sect. 2.1) and present the criteria for selection of sources for this analysis (Sect. 2.2). In Sect. 3 we explain the approach used to calculate the fluxes of the objects. The main results of this work are presented in Sect. 4 and discussed in Sect. 5. Finally, the conclusions are summarised in Sect. 6.

\section{The XMM-Newton data}

In order to carry out our analysis we used the 3491 observations included in the second XMM-Newton serendipitous source catalogue, 2XMM. The catalogue contains observations from XMM-Newton up to revolution 1338 and includes all the main MOS and pn observing modes. Only observations that were publicly available by 2007 May 01 are included in the catalogue ${ }^{2}$. 2XMM contains $246897 \mathrm{X}$-ray source detections (sources with an EPIC $0.2-12.0 \mathrm{keV}$ detection likelihood $\geq 6$ ) of which 191870 are unique X-ray sources. Extended sources comprise $\sim 8 \%$ of the detections. The data was processed with the XMM-Newton Science Analysis Software (SAS v7.1.0 ${ }^{3}$ ) and a constant pipeline configuration. This guarantees that we have a uniform data set.

\subsection{Source detection}

The source detection algorithm used to make 2XMM, eboxdetect-emldetect, is run on the three EPIC cameras and on five energy bands simultaneously: $0.2-0.5 \mathrm{keV}$, 0.5-1.0 keV, 1.0-2.0 keV, 2.0-4.5 keV and 4.5-12.0 $\mathrm{keV}^{4}$. Images, cleaned for periods of high background during the observations, are created for each camera and energy band with the SAS task evselect. For the MOS cameras events with PATTERN $\leq 12$ (single and double events) are used at all energies while for $\mathrm{pn}$, events with PATTERN $=0$ (single events) below $0.5 \mathrm{keV}$ and with PATTERN $\leq 4$ (single and double events) above $0.5 \mathrm{keV}$ are used instead. Detection masks defining the area of the detector suitable for source detection are created with the SAS task emask for each camera. Energy-dependent exposure maps are computed with the SAS task eexpmap. The eexpmap task uses the latest calibration information for the mirror vignetting, detector quantum efficiency and filter transmission to compute the telescope and instrumental throughput efficiency as a function of energy and position in the FOV. Quantum efficiency, filter transmission

\footnotetext{
1 See the on line XMM-Newton User Handbook http://xmm. esac. esa.int/external/xmm_user_support/documentation/uhb/

2 XMM-Newton started taking scientific observations in January 2000.

3 http://xmm.esac.esa.int/sas/8.0.0/

${ }^{4}$ In the $4.5-12.0 \mathrm{keV}$ band pn photons with energies between $7.8-8.2 \mathrm{keV}$ were excluded in order to avoid the instrumental background produced by $\mathrm{Cu}$ K-lines (Lumb et al. 2002).
}

and vignetting are evaluated at the mean energy of each energy band.

Initial source lists are obtained using the SAS task eboxdetect by employing a simple sliding box cell detection algorithm. Smoothed background maps for each camera and energy band are produced by the SAS task esplinemap which performs a spline fit on the source-free images, after masking out all sources detected by eboxdetect. Eboxdetect is run for a second time using the background maps produced by esplinemap, which increases the sensitivity of the source detection.

The final source lists and source parameter estimation are obtained with the SAS task emldetect. Emldetect performs a maximum likelihood fitting of the distribution of counts of sources detected by eboxdetect with the instrumental point spread function (PSF) over a circular area of 60" radius centred at the source positions. The fitting is performed using a tabulated energy and position dependent PSF. In addition, emldetect carries out a fit with the PSF convolved with a beta-model profile in order to search for sources extended in X-rays. The free parameters of the fits are the source position, count rates and extent. Position and extent are constrained to the best-fit value for all cameras and energy bands, while count rates are fitted separately for each camera and energy band. Emldetect uses the exposure maps to correct the count rates for instrumental effects (mirror vignetting, detector quantum efficiency and filter transmission). Count rates and therefore fluxes given by emldetect are background subtracted and corrected for PSF losses, i.e. they correspond to the flux in the entire PSF.

\subsection{Selection of sources}

The measured flux of a source can be significantly different between cameras for reasons other than a cross-calibration effect. For example a large discrepancy in the EPIC fluxes can be found if the source position falls in a CCD gap in one of the cameras. In order to reduce the number of "problematic" cases included in our analysis, we performed the following filtering to the source lists:

1. We used only sources detected as point-like by the source detection algorithm.

2. Sources with $<200$ background subtracted counts in the energy band of interest and on each camera were excluded. This requirement is used to avoid biases introduced by imposing a minimum value of the detection likelihood and insisting that the source is detected in both cameras (see Fig. 1).

3. Sources with off-axis angle ${ }^{5}$ greater than $12^{\prime}$ are excluded to ensure that no azimuthal biases are introduced; beyond this radius, sources begin to fall outside of the MOS CCD boundaries at various different azimuthal angles. Sources within this radius that fall on CCD gaps are also excluded if the detector coverage (weighted with the local PSF) is less than $15 \%$ (these sources are considered non-detections in the 2XMM catalogue $)^{6}$.

4. Sources with a 2.0-12.0 keV observed flux $\geq 6 \times$ $10^{-12} \mathrm{erg} \mathrm{cm}^{-2} \mathrm{~s}^{-1}$ have been excluded from the analysis as

\footnotetext{
5 The off-axis angle is the separation of the source position in the FOV from the optical axis.

6 We have checked that the use of sources with small detector coverage $(\$ 50 \%)$ has no impact on the results of our analysis.
} 


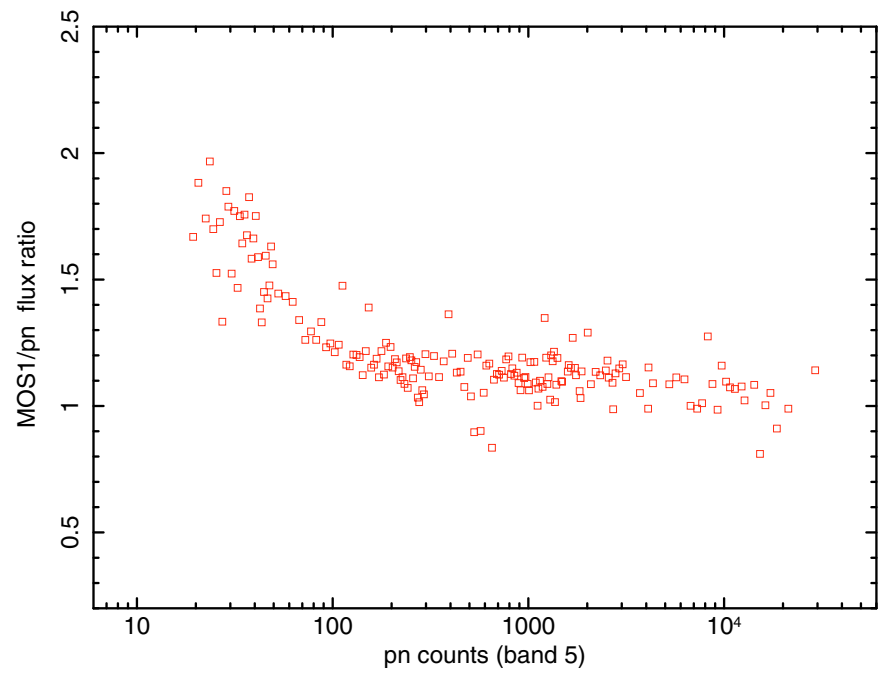

Fig. 1. MOS1 to pn flux ratio as a function of pn number of counts (background subtracted) in the $4.5-12.0 \mathrm{keV}$ energy band.

these objects suffer from pile-up ${ }^{7}$ and therefore their measured flux is underestimated.

\section{Count rate to flux conversion}

The fluxes of the sources have been computed as

Flux $=\frac{\text { RATE }}{\text { ecf }}$

where RATE are the source count rates in units of cts $\mathrm{s}^{-1}$ and ec $f$ are the energy conversion factors from count rates to fluxes, in units of cts $\mathrm{cm}^{2} \mathrm{erg}^{-1}$. Count rates are background subtracted and are corrected for PSF losses and variations of the effective exposure across the EPIC FOV.

The energy conversion factors are different for each EPIC camera and depend on the optical blocking filter used for the observation and the observing mode ${ }^{8}$. The dependence of the energy conversion factors on the observing mode is small $(1.0-2.0 \%)$, hence the ecf values have only been computed for the PrimeFullWindow mode, which is the observing mode most frequently used for XMM-Newton observations. The ecf values have been computed for each EPIC camera, energy band and optical blocking filter.

In order to calculate the energy conversion factors it is necessary to assume a spectral model. In the case of 2XMM and also for this work we have assumed that the model that best reproduces the broad band X-ray emission of the sources is a power-law with photon index $\Gamma=1.7$ and observed absorption $N_{\mathrm{H}}=3 \times 10^{20} \mathrm{~cm}^{-2}$. This model has been found to be a good representation of the spectra of the bulk of 2XMM sources

\footnotetext{
7 If a source is bright enough, two or more X-ray photons might deposit their charge in a single pixel or in neighbouring pixels during one read-out cycle. This is known as pile-up. In such a case these events are recognised as one single event with an energy equivalent to the sum of the contributing photon energies. Pile-up reduces the counts in the central part of the source, resulting in flux loss.

${ }^{8}$ Each EPIC camera is equipped with a set of three separate filters, named thick, medium and thin. For a detailed description of the science modes of the EPIC cameras see the on line XMM-Newton User Handbook http://xmm.esac.esa.int/ external/xmm_user_support/documentation/uhb/
}

(Watson et al. 2008). Note that the fluxes have not been corrected for the Galactic absorption along the line of sight, and therefore they are absorbed fluxes.

Since the release of 2XMM there have been important updates on the calibration information of the EPIC cameras. For example, for the pn, new versions of the public redistribution matrix files ( $\mathrm{rmf}$ ) are available. The most recent public calibration files ${ }^{9}$ available at the time of writing, were used to create matrices used in the computation of the energy conversion factors. These differ from the EPIC calibration files used in the pipeline processing of the 2XMM catalogue. Note that correction factors to apply to the 2XMM fluxes are provided in the on-line documentation of the catalogue $^{10}$. As indicated in Sect. 2.1, the event selection used in the 2XMM pipeline is for the pn detector: PATTERN $\leq 4$ at energies above $0.5 \mathrm{keV}$ and PATTERN $=0$ only at energies below $0.5 \mathrm{keV}$. For the MOS cameras PATTERN $\leq 12$ selection is made at all energies. Therefore, for the EPIC pn detector we used the PrimeFullWindow mode on-axis redistribution matrices for singles only (for the energy band $0.2-0.5 \mathrm{keV}$ ) and singles plus doubles (for the energy bands above $0.5 \mathrm{keV}$ ). The fluxes given by the EPIC pn camera from the new calibration are overall $2 \%$ lower than 2XMM fluxes at energies below $0.5 \mathrm{keV}$ while at higher energies the EPIC pn flux changes are $<1 \%$ (see calibration notes XMM-CCF-REL-189, XMM-CCF-REL-205).

For the MOS cameras new quantum efficiency files (see XMM-CCF-REL-235) have become available since the release of 2XMM. In addition, a significant change in the MOS low energy redistribution characteristics with time has been confirmed. This effect is important only for sources with a separation from the optical axis $\$ 2^{\prime}$, which in most cases is the target of the observation (Read et al. 2006). To account for this effect, epochdependent MOS redistribution matrices have been generated for use by observers (XMM-CCF-REL-202). However for this work we have followed the approach of 2 XMM and used an average response to compute the MOS energy conversion factors. In order to investigate the EPIC flux cross-calibration using the latest calibration available we computed new MOS redistribution matrices more representative of the current calibration than the ones used in 2XMM. The MOS1 and MOS2 response matrices were computed with the SAS task rmfgen for revolution 375, on-axis, and for PATTERNs from 0-12. These changes in the MOS calibration have resulted in an increase of MOS fluxes compared to the quoted values in 2 XMM by $\sim 5-8 \%$ in the $0.2-0.5 \mathrm{keV}$ band, $\sim 4 \%$ in the $0.5-1.0 \mathrm{keV}$ energy band and $\sim 1 \%$ from $1.0-2.0 \mathrm{keV}$. At higher energies the changes in MOS flux are negligible. An investigation of the EPIC flux cross-calibration using MOS epoch and position dependent responses is beyond the scope of this paper. However as we will see in Sect. 5 the use of an "average" MOS response for all sources has a small effect on the main conclusions of this work as the time dependent effect only affects a small fraction of the objects involved in our analysis and at low energies only (below $\sim 0.5 \mathrm{keV}$ ).

On-axis MOS and pn effective area files were produced by the SAS task arfgen. The count rates from emldetect are corrected for the effective exposure along the FOV (which includes vignetting and bad pixel corrections) and the PSF enclosed energy fraction. Hence the effective areas were generated by disabling these corrections, as indicated in the documentation of the SAS task arfgen. MOS and pn effective area curves for the

\footnotetext{
9 http://xmm2.esac.esa.int/external/xmm_sw_cal/calib/ epic_files.shtml

10 http://xmmssc-www.star.le.ac.uk/Catalogue/ xcat_public_2XMM.html
} 
Table 1. Count rate to flux energy conversion factors (ecf) ${ }^{a}$.

\begin{tabular}{|c|c|c|c|c|c|c|c|c|c|c|c|c|}
\hline \multirow{2}{*}{$\begin{array}{c}\text { Energy band } \\
\text { keV }\end{array}$} & \multicolumn{3}{|c|}{ Open } & \multicolumn{3}{|c|}{ Thin } & \multicolumn{3}{|c|}{ Medium } & \multicolumn{3}{|c|}{ Thick } \\
\hline & $e c f_{p n}$ & $\mathrm{ecf}_{\mathrm{m} 1}$ & $e c f_{\mathrm{m} 2}$ & $e c f_{\mathrm{pn}}$ & $\mathrm{ecf}_{\mathrm{m} 1}$ & $e c f_{\mathrm{m} 2}$ & $e c f_{p n}$ & $\mathrm{ecf}_{\mathrm{m} 1}$ & $e c f_{\mathrm{m} 2}$ & $e c f_{\mathrm{pn}}$ & $e c f_{\mathrm{m} 1}$ & $\mathrm{ecf}_{\mathrm{m} 2}$ \\
\hline $0.2-0.5$ & 16.530 & 3.094 & 3.142 & 9.056 & 1.725 & 1.738 & 7.880 & 1.514 & 1.521 & 4.699 & 0.985 & 0.983 \\
\hline $0.5-1.0$ & 10.256 & 2.191 & 2.196 & 8.253 & 1.807 & 1.810 & 7.995 & 1.756 & 1.7 & 6.138 & 1.426 & 1.428 \\
\hline $1.0-2.0$ & 6.166 & 2.125 & 2.132 & 5.878 & 2.028 & 2.034 & 5.779 & 1.993 & 1.999 & 4.999 & 1.775 & 1.781 \\
\hline $2.0-4.5$ & 1.982 & 0.758 & 0.762 & 1.950 & 0.747 & 0.751 & 1.927 & 0.738 & 0.742 & 1.827 & 0.708 & 0.712 \\
\hline $4.5-12.0$ & 0.556 & 0.144 & 0.151 & 0.555 & 0.144 & 0.151 & 0.554 & 0.143 & 0.151 & 0.548 & 0.141 & 0.149 \\
\hline
\end{tabular}

${ }^{a}$ Energy conversion factors have been calculated for each camera, optical blocking filter of the observation and energy band. To obtain the values a power-law spectral model of photon index $\Gamma=1.7$ and observed absorption $N_{\mathrm{H}}=3 \times 10^{20} \mathrm{~cm}^{-2}$ was assumed. The ecf are given in units of $10^{11} \mathrm{cts} \mathrm{cm}^{2} \mathrm{erg}^{-1}$.

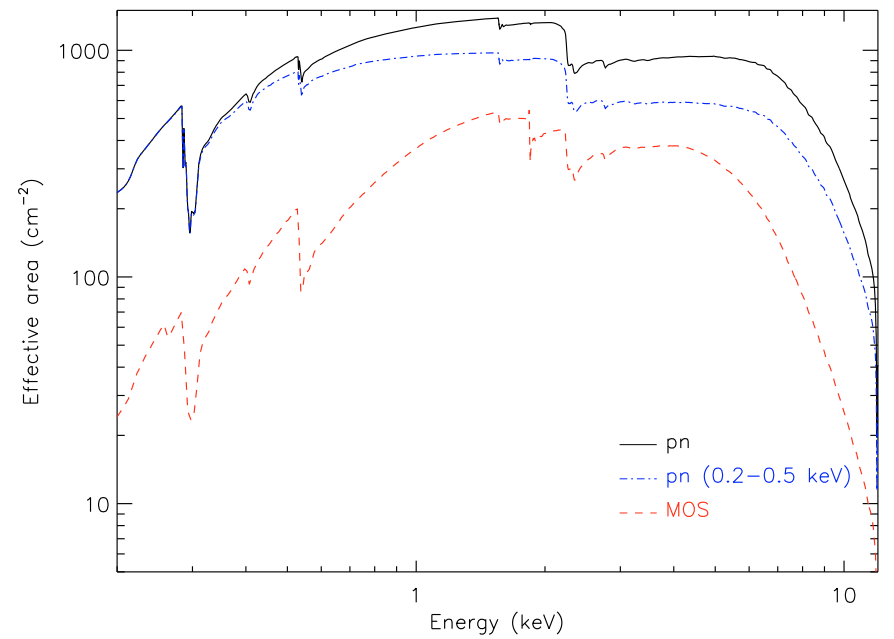

Fig. 2. Variation of the effective area of the EPIC cameras (for the medium filter) as a function of energy. The dot-dashed and solid lines show the effective area for the EPIC pn camera, for single pixel events (used below $0.5 \mathrm{keV}$ ) and for single+double pixel events (used above $0.5 \mathrm{keV}$ ) respectively. The dashed line shows the effective area for the EPIC MOS cameras.

medium filter are shown in Fig. 2. For pn we show the effective area curves used to compute ecf values both above and below $0.5 \mathrm{keV}$. The energy conversion factors are listed in Table 1.

\section{Results}

In order to quantify the systematic difference in flux between the EPIC cameras we have performed a statistical analysis, comparing the flux ratio for large samples of sources selected as specified in Sect. 2. We computed for each source the value $\left(S_{i}-S_{j}\right) / S_{j}$ where $S_{i}$ and $S_{j}$ are the fluxes of the source measured by cameras $(i, j)$ respectively.

The obtained distributions were fitted with a Gaussian profile, which, thanks to the large number of objects involved in the analysis, provided in all cases a good enough representation of the distribution of values.

\subsection{Energy dependence}

Figures 3 and 4 show the distributions of difference in flux (in percentage units) when comparing pn and MOS cameras for sources detected in different energy bands. The results of the comparison between pn and MOS2 are very similar to those obtained for the pn and MOS1 cameras therefore we only show the latter. The smooth solid lines show the $\chi^{2}$ best-fit Gaussian distributions of the data. The best-fit parameters and number of sources involved in the analysis are shown in the upper-left corner of the plots and are listed in Table 2. Figure 5 shows the systematic difference in the measured flux between cameras as a function of energy.

The mean flux difference between the MOS and pn cameras is less than $10 \%$ at energies $\leq 4.5 \mathrm{keV}$ and $\sim 10-13 \%$ above $4.5 \mathrm{keV}$. In all but the very lowest energy cases MOS fluxes are found to be higher than those reported for the pn camera. The relative flux calibration between the two MOS cameras is better than $4 \%$ at all energies sampled by our analysis.

In order to obtain the conversion factors from count rate to flux we assumed the same spectral model for all sources. However we know that the sources that populate the X-ray sky have a broad range of spectral shapes. For example, the X-ray emission of AGN often shows more spectral complexity than a simple power law (excess X-ray absorption, soft excess). Indeed even the distribution of broad band continuum shapes is known to have an intrinsic dispersion $\Delta \Gamma \sim 0.2-0.3$ (see e.g. Mateos et al. 2005). Furthermore, at soft $X$-ray energies and bright fluxes the contribution from non-AGN populations to the X-ray sky, mainly stars and clusters of galaxies with thermal spectra, is not negligible (Mateos et al. 2008). We have investigated the impact of the variety of spectral shapes on the flux cross-calibration of the EPIC cameras. In order to quantify the impact of the chosen spectral model on the measured EPIC flux cross-calibration we have computed the change in the flux ratio between cameras for a varying power-law spectral model. We performed the calculation by varying the continuum shape up to $\Delta \Gamma=0.6$. Our simulations have shown that the chosen spectral model has a small effect on the measured flux from $0.5 \mathrm{keV}$ to $4.5 \mathrm{keV}$ where a $\Delta \Gamma=$ 0.6 variation changes the measured flux by less than $4 \%$. The EPIC MOS and pn fluxes have a much stronger dependence on the spectral model used to compute the ecf in the $0.2-0.5 \mathrm{keV}$ and 4.5-12.0 keV energy bands where a $\Delta \Gamma=0.6$ can vary the measured fluxes by $\sim 15 \%$. While the spectral model has an important effect on the measured flux, the impact on the flux ratio between cameras is small. In order to confirm this result we have also computed the flux ratio distributions from the sub-set of sources whose hardness ratios are consistent with the spectral model assumed to calculate the ecf. We found that the results were largely indistinguishable from the "full set" of sources, thus confirming that our approach of using a fixed spectral model to compute source fluxes has a small impact on the main results of our analysis.

\subsection{Time dependence}

Here we present the dependence of the flux cross-calibration of the EPIC cameras on the lifetime of the mission. To do that we 

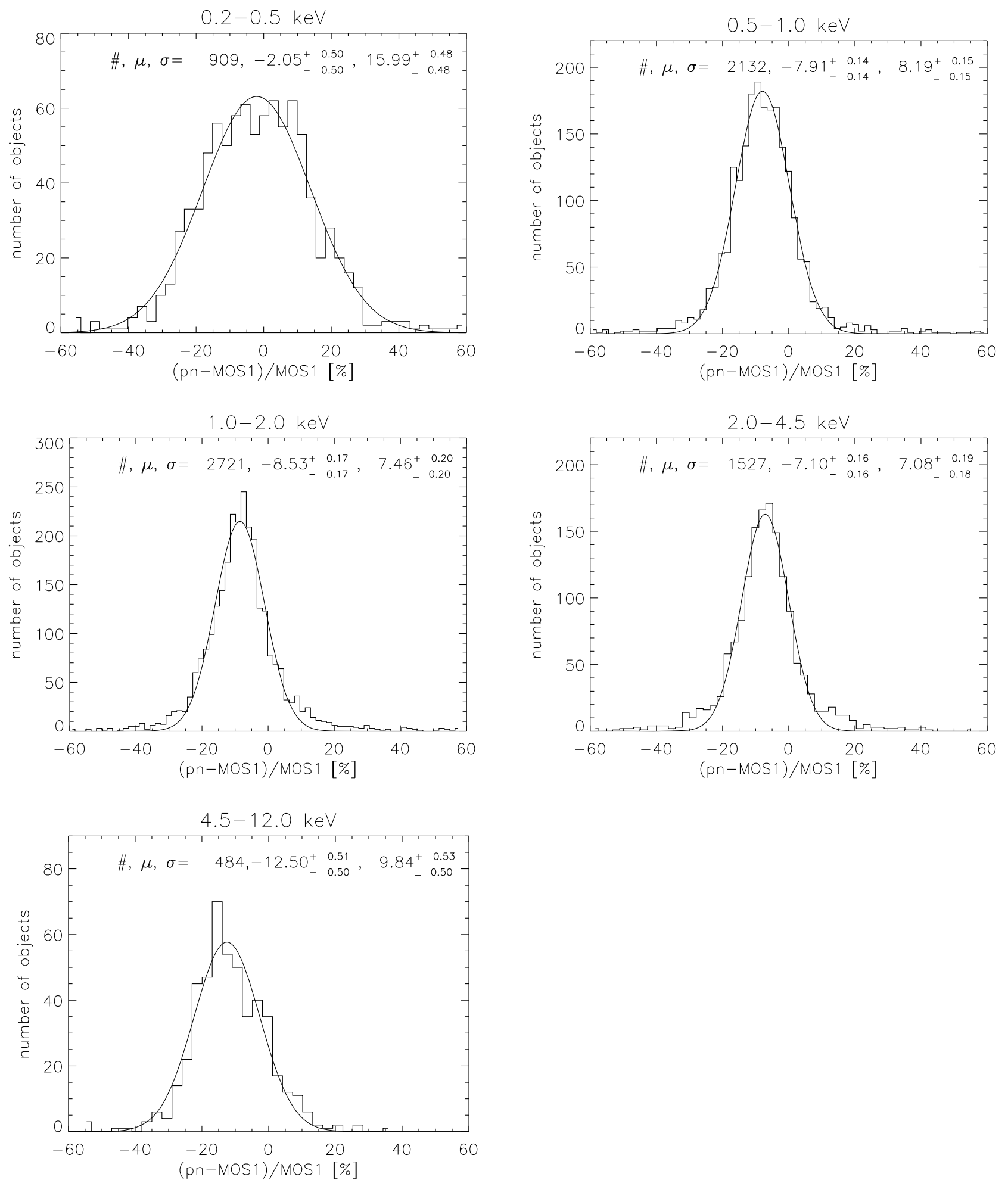

Fig. 3. Distributions of the percentage flux difference between the EPIC pn and MOS1 cameras as a function of the energy band. The best-fit of the experimental distributions with a Gaussian function is shown with a smooth solid line and the corresponding best-fit parameters (mean and dispersion) and number of sources involved in the analysis are listed in the upper-left corner of the plots (also given in Table 2). Errors are $90 \%$ confidence.

have computed the distributions of EPIC flux ratios for sources detected at different epochs. We have grouped our sources in five XMM-Newton revolution intervals of the same size to have a uniform sampling of the lifetime of the mission. The grouping definition is shown in Table 3 while the observed time dependence of the flux cross-calibration of the EPIC cameras at different energies is shown in Fig. 6.

The flux cross-calibration between the MOS cameras does not depend significantly on time and the result seems to hold at all energies. When comparing pn and MOS fluxes, no 

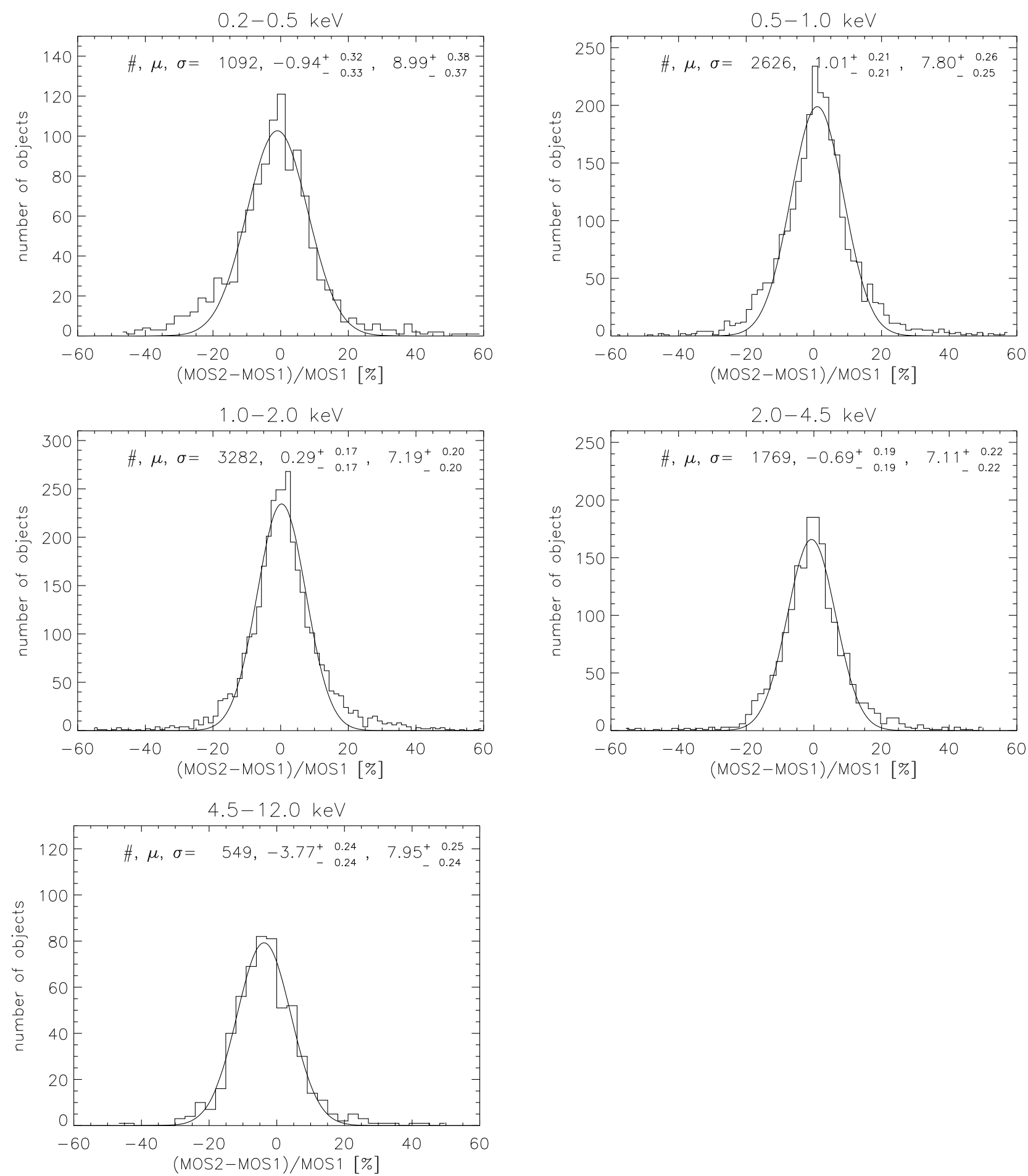

Fig. 4. Distributions of the percentage flux difference between the EPIC MOS1 and MOS2 cameras as a function of the energy band. The best-fit of the experimental distributions with a Gaussian function is shown with a smooth solid line and the corresponding best-fit parameters (mean and dispersion) and number of sources involved in the analysis are listed in the upper-left corner of the plots (also given in Table 2). Errors are $90 \%$ confidence.

significant change of the flux ratio with time is seen at energies above $0.5 \mathrm{keV}$. However, a strong temporal dependence of the flux ratio is found in the $0.2-0.5 \mathrm{keV}$ band, in the sense that pn fluxes seem to increase with respect to MOS fluxes at higher revolutions. The effect is more important when comparing pn and MOS2 fluxes. We note that there is some scatter in the pn vs. MOS1 values obtained at different epochs at energies above $4.5 \mathrm{keV}$ due to low statistics, although the points are consistent at $2-3 \sigma$.

\subsection{Dependence on position of sources in the FOV}

It is well known that the response of the XMM-Newton mirrors and the EPIC instruments varies with position in the FOV. Here we investigate the relative flux cross-calibration of the EPIC cameras, by comparing the EPIC fluxes for sources at different off-axis and azimuthal angles. 
Table 2. Summary of the statistical comparison of EPIC fluxes in different energy bands.

\begin{tabular}{|c|c|c|c|c|c|c|c|c|c|}
\hline \multirow{2}{*}{$\begin{array}{c}\text { Energy band } \\
(\mathrm{keV})\end{array}$} & \multicolumn{3}{|c|}{ pn vs. MOS1 } & \multicolumn{3}{|c|}{ pn vs. MOS2 } & \multicolumn{3}{|c|}{ MOS2 vs. MOS1 } \\
\hline & $\mu(\%)$ & $\sigma$ & $N_{\text {srcs }}$ & $\mu(\%)$ & $\sigma$ & $N_{\text {srcs }}$ & $\mu(\%)$ & $\sigma$ & $N_{\text {srcs }}$ \\
\hline $0.2-0.5$ & $-2.0 \pm 0.5$ & $16.0 \pm 0.5$ & 909 & $1.2 \pm 0.4$ & $13.2 \pm 0.4$ & 885 & $-0.9 \pm 0.3$ & $9.0 \pm 0.4$ & 1092 \\
\hline $0.5-1.0$ & $-7.9 \pm 0.1$ & $8.2 \pm 0.2$ & 2132 & $-8.2 \pm 0.1$ & $7.9 \pm 0.2$ & 2197 & $1.0 \pm$ & $7.8 \pm 0.3$ & 2626 \\
\hline $1.0-2.0$ & $-8.5 \pm 0.2$ & $7.5 \pm 0.2$ & 2721 & $-9.1 \pm 0.2$ & $7.3 \pm 0.2$ & 2781 & $0.3 \pm 0.2$ & $7.2 \pm 0.2$ & 3282 \\
\hline $2.0-4.5$ & $-7.1 \pm 0.2$ & $7.1 \pm 0.2$ & 1527 & $-6.5 \pm 0.2$ & $7.4 \pm 0.2$ & 1546 & $-0.7 \pm 0.2$ & $7.1 \pm 0.2$ & 1769 \\
\hline $4.5-12.0$ & $-12.5 \pm 0.5$ & $9.8 \pm 0.5$ & 484 & $-9.0 \pm 0.5$ & $9.5 \pm 0.5$ & 502 & $-3.8 \pm 0.2$ & $8.0 \pm 0.2$ & 549 \\
\hline
\end{tabular}

$\mu$ and $\sigma$ are the $\chi^{2}$ best-fit mean and dispersion of the Gaussian distribution used to fit the data. $N_{\text {srcs }}$ is the total number of sources involved in the analysis. Errors are $90 \%$ confidence.

Table 3. Grouping definition used to study the time dependence of the EPIC flux cross-calibration.

\begin{tabular}{cc}
\hline \hline Revolution $^{a}$ & $N_{\text {obs }}^{b}$ \\
\hline $28-290$ & 730 \\
$291-552$ & 919 \\
$553-814$ & 901 \\
$815-1076$ & 739 \\
$1077-1338$ & 202 \\
\hline
\end{tabular}

${ }^{a}$ Range of XMM-Newton revolutions in group; ${ }^{b}$ total number of observations with revolution in group.

The variation of the percentage flux difference between the EPIC pn and MOS cameras as a function of energy band for sources at different MOS off-axis angles is shown in Fig. 7. Here sources have been divided into groups with MOS off-axis angles of $0^{\prime}-2^{\prime}, 2^{\prime}-5^{\prime}, 5^{\prime}-8^{\prime}$ and $8^{\prime}-12^{\prime}$.

There is a clear trend for sources further from the optical axis to show an increased flux in the MOS cameras relative to pn. This is particularly apparent for MOS1. In the lowest energy band the on-axis sources show a significant excess of pn flux relative to the MOS. This is a very different result than obtained for sources at larger off-axis angles. In both MOS cameras, in the highest energy band the MOS flux excess increases by $10-15 \%$ as the off-axis angle increases. This highly significant result is likely to be due to inaccuracies in the calibration of either the vignetting, the PSF or the RGA obscuration. As a further diagnostic we have produced samples of sources with a MOS off-axis angle $>2$ ' (to reduce the effect due to the change in the low energy redistribution characteristics of the MOS cameras with time, see Sect. 3) and then divided into four different azimuthal angle bins. The results are shown in Fig. 8. For both MOS cameras, the positional flags indicate the quadrants delineated by a diagonal cross centred on $\mathrm{CCD}=1$, RAWX $=300$, RAWY $=300$, such that flag $=1$ extends towards the low RGA obscuration (i.e. high MOS throughput) direction, and flag $=3$ extends towards the high RGA obscuration (i.e. low MOS throughput) direction, as shown in Fig. 9.

In the 4.5-12.0 keV band there is a very strong dependence of the MOS to pn flux ratio on the azimuthal angle. For both MOS cameras, sources lying along the RGA dispersion axis show a large gradient in MOS vs. pn relative flux.

\section{Discussion}

The results for the full source sample show an excellent agreement between the two MOS cameras; better than $4 \%$ over the entire energy range of the cameras. The MOS cameras present a consistent excess in flux compared with the pn of $\sim 7-9 \%$ from $0.5 \mathrm{keV}$ to $4.5 \mathrm{keV}$ and $\sim 10-13 \%$ at higher energies $(\gtrsim 4.5 \mathrm{keV})$.
These results are in agreement with the findings of Stuhlinger et al. (2008), who performed a careful analysis of 168 bright onaxis sources.

At low energies $(0.2-0.5 \mathrm{keV})$ the agreement between the EPIC cameras is seemingly better $(<3 \%)$. However, a strong trend in the flux ratios with observation epoch, in this band, is evident as shown in Fig. 6. This can be explained by the evolution of the MOS redistribution characteristics with time. In this analysis we have used a single response matrix for each of the MOS cameras, relevant for an on-axis source observed in revolution 375, which will necessarily be inaccurate for observations made at earlier and later epochs. The redistribution function evolves such that an increasing fraction of X-rays suffer incomplete charge collection. The effect is increasingly stronger towards lower energies. The evolution of the MOS rmf seems to be related to the total $\mathrm{X}$-ray radiation dose received by a pixel which will clearly be higher at the centre of the detector where bright sources are preferentially placed (Read et al. 2006). Sources at MOS off-axis angles greater than $\sim 2^{\prime}$ are essentially immune to this effect.

For MOS on-axis sources, photons from the energy band $0.2-0.5 \mathrm{keV}$ can be redistributed in energy below the detection threshold and lost while photons from energy band $0.5-1.0 \mathrm{keV}$ can be redistributed into the $0.2-0.5 \mathrm{keV}$ energy band. The effect is negligible at energies above $\sim 1 \mathrm{keV}$. For a given source, the strongest evolution in observed flux occurs in the $0.2-0.5 \mathrm{keV}$ band and is dependent upon the intrinsic spectrum. Highly absorbed sources which have a very low $0.2-0.5 \mathrm{keV}$ to $0.5-1.0 \mathrm{keV}$ ratio will show an increase in $0.2-0.5 \mathrm{keV}$ flux as more photons are gained from the $0.5-1.0 \mathrm{keV}$ band than are lost.

The majority of sources in this sample, however, have relatively soft spectra, for example AGN with low absorption, and the evolution of the rmf causes a loss of $0.2-0.5 \mathrm{keV}$ flux as more photons are lost below threshold than gained from the $0.5-1.0 \mathrm{keV}$ energy band. This is confirmed by Fig. 7 where the $0.2-0.5 \mathrm{keV}$ ratio at small off-axis angles is significantly different from the other points. We have checked that the distribution of off-axis angles of the sources are very similar at all epochs used in our analysis, therefore the time dependence observed in the MOS vs. pn 0.2-0.5 keV flux ratios (see Fig. 6) cannot be explained as an artificial effect due to the increasing fraction of near on-axis sources with time.

In Fig. 8 we see that, once the sources from the innermost $2^{\prime}$ are excluded from the analysis, the $0.2-0.5 \mathrm{keV}$ flux ratios show a spread of up to $\sim 8 \%$ with the detector azimuthal angle. As this flux ratio is mostly strongly influenced by the calibration of the detector rmfs we suspect that this spread is due to intrinsic CCD to $\mathrm{CCD}$ variations in the low energy response of the $14 \mathrm{MOS}$ and 12 pn CCDs. The quality of the ground calibration data below $500 \mathrm{eV}$ is not sufficient to discriminate between different 

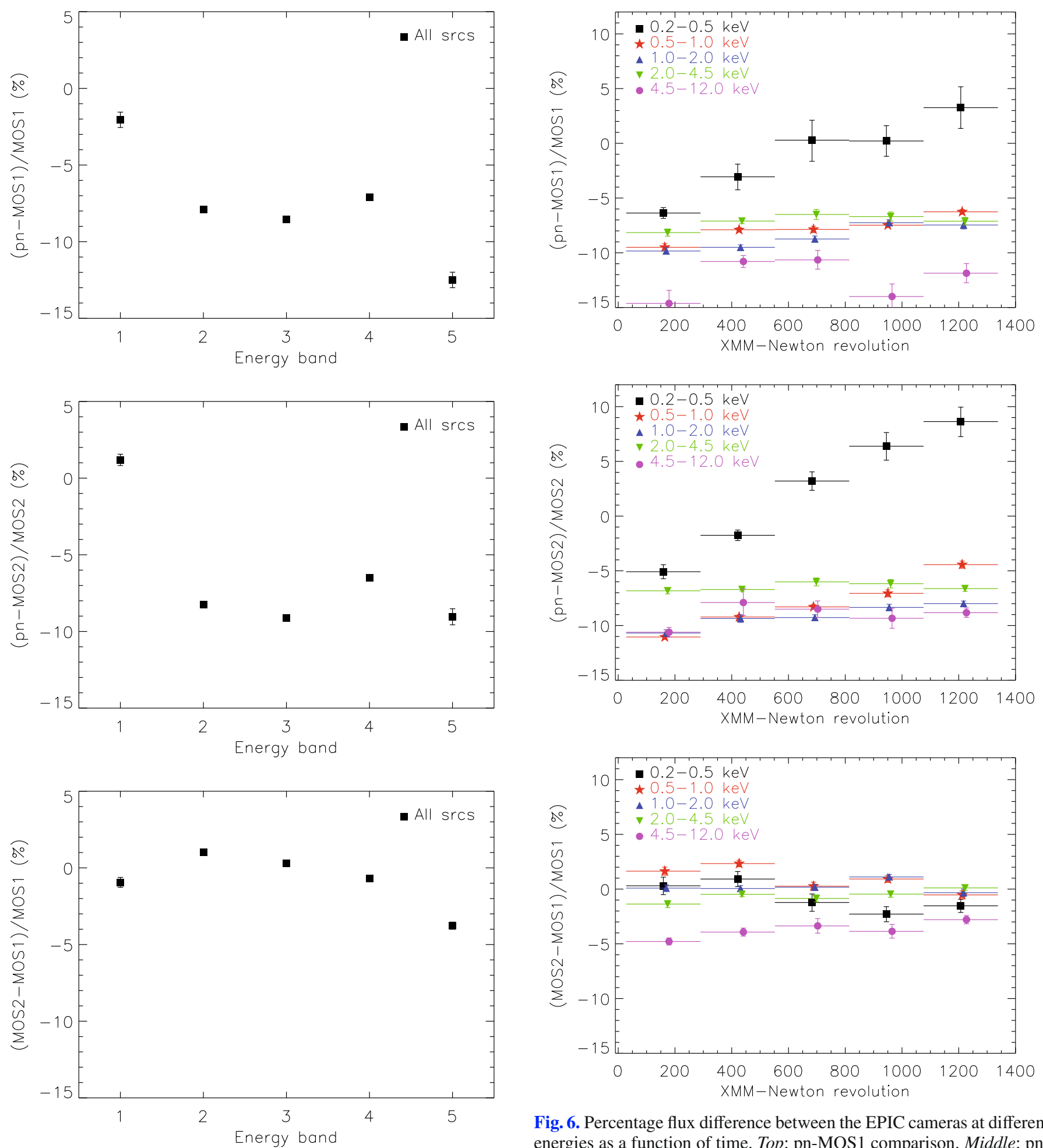

Fig. 6. Percentage flux difference between the EPIC cameras at different energies as a function of time. Top: pn-MOS1 comparison. Middle: pnMOS2 comparison. Bottom: MOS2-MOS1 comparison. Errors are $90 \%$

Fig. 5. Mean percentage flux difference between the EPIC cameras as a function of energy. The $x$-axis shows the energy band identification number used in this work: $1: 0.2-0.5 \mathrm{keV}, 2: 0.5-1.0 \mathrm{keV}, 3$ : 1.0-2.0 keV, 4: 2.0-4.5 keV and 5: 4.5-12.0 keV. Top: pn-MOS1 comparison. Middle: pn-MOS2 comparison. Bottom: MOS2-MOS1 comparison. Errors are $90 \%$ confidence.

devices (XMM-EPIC instrument team, private communication) so we cannot compare our results directly with a prediction based on the available calibration data. Further progress on this will require the accumulation of sufficient numbers of sources

to enable comparisons to be made on specific combinations of CCDs.

Excluding sources from the innermost $2^{\prime}$, the $0.2-0.5 \mathrm{keV}$ flux ratios are $-11.4 \pm 0.3 \%,-4.6 \pm 0.6 \%$ and $-4.8 \pm 0.7 \%$ for the pn/MOS1, pn/MOS2 and MOS2/MOS1 ratios respectively bringing them into better agreement with the values at energies from $0.5 \mathrm{keV}$ to $4.5 \mathrm{keV}$. It is clear from this that the use of a single MOS rmf to calculate the global flux ratios at the lowest energies is not correct. 

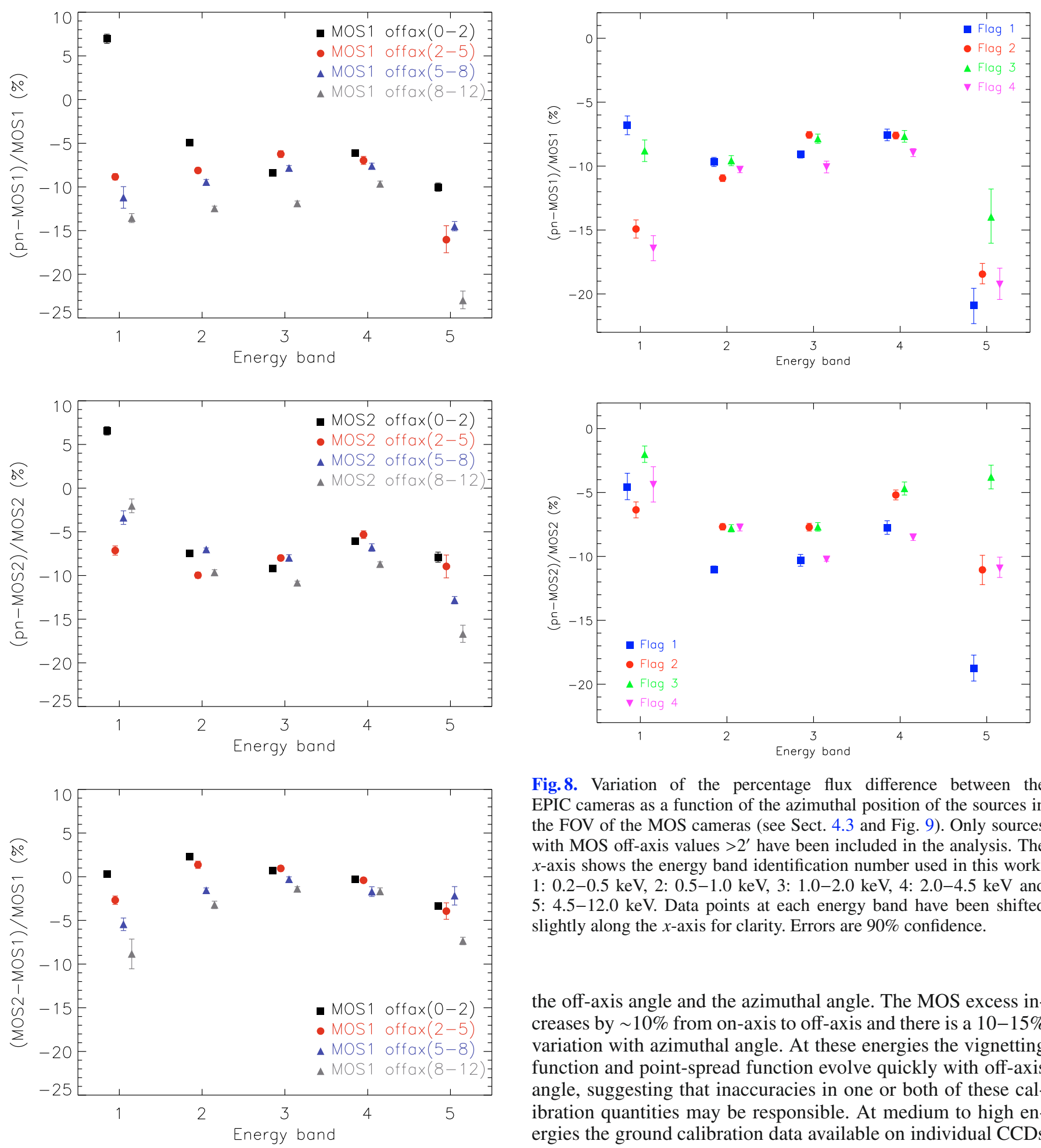

Fig. 8. Variation of the percentage flux difference between the EPIC cameras as a function of the azimuthal position of the sources in the FOV of the MOS cameras (see Sect. 4.3 and Fig. 9). Only sources with MOS off-axis values $>2^{\prime}$ have been included in the analysis. The $x$-axis shows the energy band identification number used in this work: 1: $0.2-0.5 \mathrm{keV}, 2: 0.5-1.0 \mathrm{keV}, 3: 1.0-2.0 \mathrm{keV}, 4: 2.0-4.5 \mathrm{keV}$ and 5: $4.5-12.0 \mathrm{keV}$. Data points at each energy band have been shifted slightly along the $x$-axis for clarity. Errors are $90 \%$ confidence.

the off-axis angle and the azimuthal angle. The MOS excess increases by $\sim 10 \%$ from on-axis to off-axis and there is a $10-15 \%$ variation with azimuthal angle. At these energies the vignetting function and point-spread function evolve quickly with off-axis angle, suggesting that inaccuracies in one or both of these calibration quantities may be responsible. At medium to high energies the ground calibration data available on individual CCDs suggests that $\mathrm{CCD}$ to $\mathrm{CCD}$ variations in detector response are not responsible for the apparent azimuthal dependence seen in the 4.5-12.0 keV band in Fig. 8. For example, the spread in the measured quantum efficiency of the MOS CCDs is less than 5\% from 4.5 to $6.5 \mathrm{keV}$ and is too small to account for the $15 \%$ variation seen in the pn to MOS2 flux ratio in the $4.5-12.0 \mathrm{keV}$ band. In principle the pn camera has no azimuthal dependence for any of the calibration quantities, whereas the vignetting of the MOS cameras is strongly affected by the transmission of the RGS gratings (Turner et al. 2001). Furthermore, because the outer MOS chips lie on two distinct planes, it is believed that small $<5 \%$ variations due to the PSF may exist (Saxton et al. 2003). 

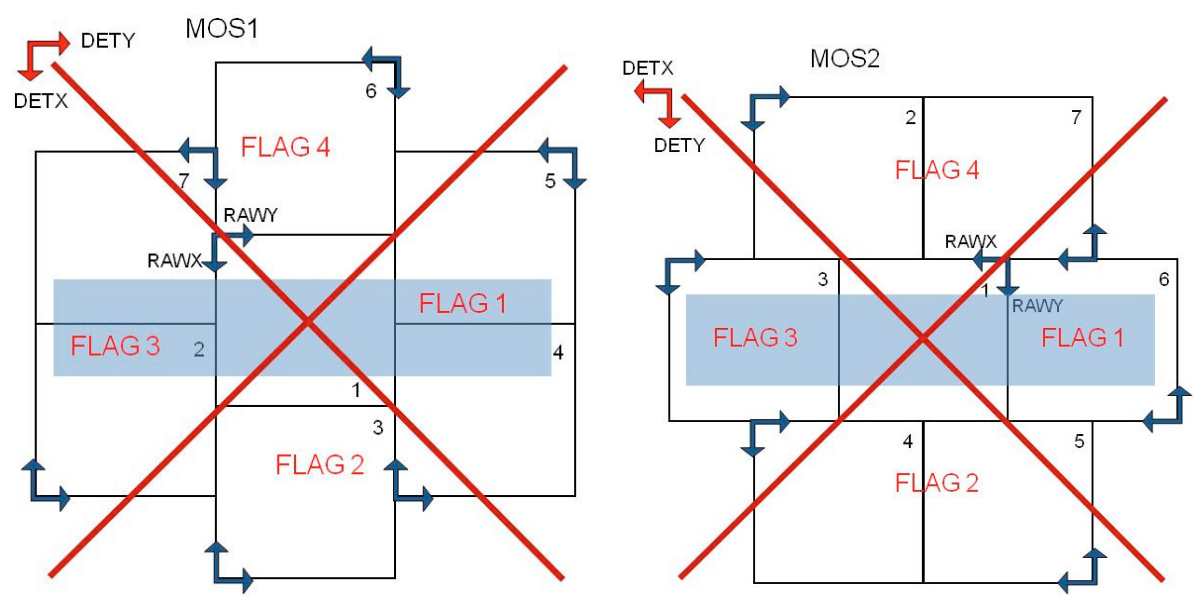

Fig. 9. MOS1 and MOS2 CCD layouts and CCD numbering (small numbers on the top right corner of the CCDs). The position of the RGSs is indicated with a shaded rectangle. The diagonal cross and large "FLAG n" text indicate the positional flag settings. The MOS diagrams are co-aligned such that the individual sources occupy the same position in each diagram e.g. a source in the middle of MOS1 CCD 6, lies in the middle of the $\mathrm{CCD} 2 / \mathrm{CCD} 7$ boundary in MOS2.

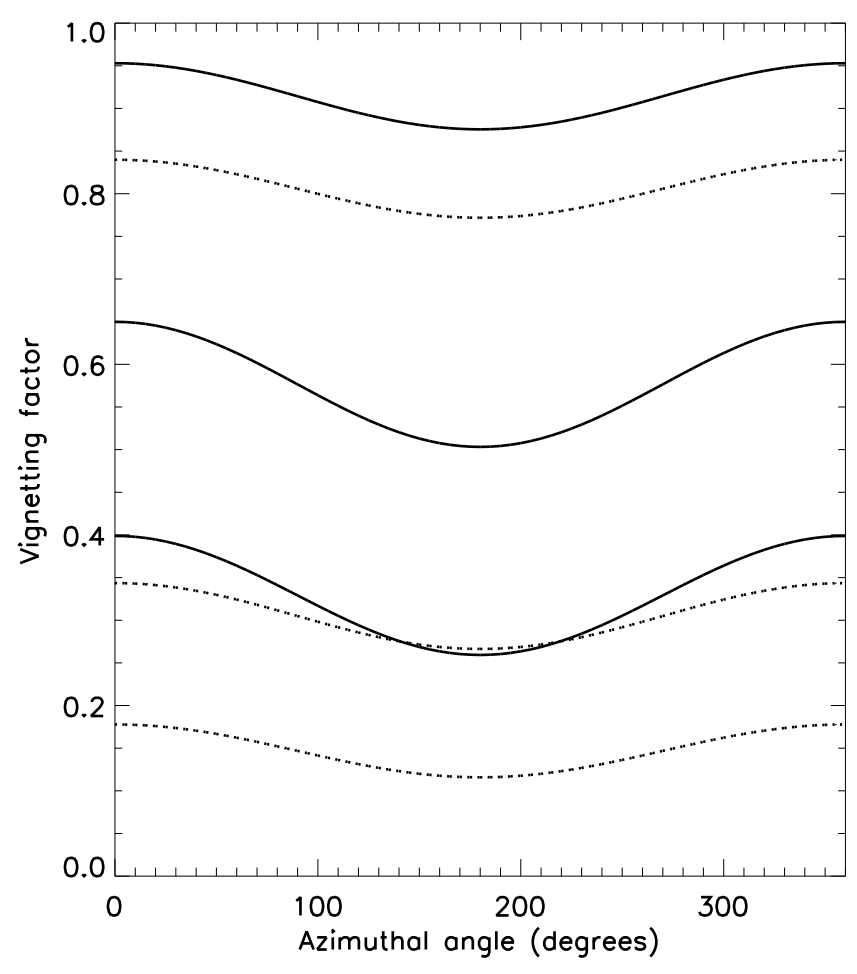

Fig. 10. MOS vignetting factor as a function of azimuthal angle at energies of $1 \mathrm{keV}$ (solid lines) and $10 \mathrm{keV}$ (dotted lines), and for off-axis angles of (top to bottom) $3^{\prime}, 9^{\prime}$ and $15^{\prime}$.

In Fig. 10 we show the current calibration of the MOS vignetting function for off-axis angles of $3^{\prime}, 9^{\prime}$ and $15^{\prime}$ as a function of the azimuthal angle. The azimuthal dependency, due to obscuration by the RGA structure, is both energy and offaxis angle dependent. At $10 \mathrm{keV}$ and $9^{\prime}$ off-axis it drops by $25 \%$ between the high MOS throughput direction (azimuth = $0^{\circ}$; MOS flag 1 in Figs. 8 and 9) and the opposite direction (azimuth $=180^{\circ}$; MOS flag 3 in Figs. 8 and 9). Therefore the results presented in Fig. 8 are highly suggestive that a calibration change is required: increasing the magnitude of the azimuthal vignetting variation at high energies would bring the points in Fig. 8 closer together. Furthermore, a change in the radial vignetting calibration to increase the overall MOS throughput at the highest energies might also be required. This is especially the case for MOS1 where a large overall offset at high energies is seen in Fig. 5.
Though the effect introduced by the outer MOS chips lying on two distinct planes is thought to be small, it can be examined in the present analysis. The outer MOS chips run up-down, up-down around the central chip, such that the positional flag settings used in Figs. 8 and 9 divide MOS1 into two flags (2 and 4) that are dominated by an "up" chip and by a "down" chip, and are free from the gross effects of the azimuthal vignetting (at azimuth $=0^{\circ}$ and azimuth $=180^{\circ}$ ). The situation is not as good for MOS2 as, although CCDs 6 and 3 can be compared by comparing flags 1 and 3, these positions suffer the largest deviations in azimuthal vignetting due to the RGA obscuration. For MOS1 at least though we can examine the MOS chip plane PSF effect on the obtained source fluxes: the flux ratio values in these two areas (MOS1, flags 2 and 4) in Fig. 8 are seen to agree with each other very well (within $2 \%$ ), indicating that the effect on the outer MOS chips lying on two distinct planes is very small.

\section{Conclusions}

We have used the second XMM-Newton serendipitous source catalogue, 2XMM, to compile large samples of "good quality" sources to measure the difference in flux seen by the EPIC cameras and investigate the relative calibration of the instruments. The main results are given below.

1. The MOS cameras agree with each other to better than $4 \%$ at all energies.

2. The MOS cameras register a consistent $7-9 \%$ higher flux than the pn at energies between $0.5 \mathrm{keV}$ and $4.5 \mathrm{keV}$.

3. The pn/MOS flux discrepancy increases at high energies, up to $\sim 13 \%$ for MOS1. The flux discrepancy at high energies has been shown to have a strong off-axis and azimuthal angle dependence. The most likely explanation for this variation is an incorrect calibration of the obscuration factor in the MOS cameras due to the RGA structures. As the azimuthal variation is not present at energies below $4.5 \mathrm{keV}$, a reworking of the RGA physical parameters is needed which solves the high energy problem without affecting lower energies. If this can be achieved then the mirror vignetting function for the EPIC MOS cameras will need to be revisited as these two quantities are intimately related. Ideally, the solution would reduce the overall discrepancy of the cameras at higher energies and leave a uniform MOS/pn excess at all energies.

4. A systematic difference in flux cross-calibration is seen for the $0.2-0.5 \mathrm{keV}$ band with a strong dependence on time. This effect, also present in the 2XMM catalogue, has been 
explained as being due to the fact that an average response has been used to compute the fluxes of sources detected with the MOS camera at all epochs. Therefore this effect could be removed by using an epoch and position dependent response function to compute the MOS1 and MOS2 fluxes and could be used in future releases of XMM-Newton catalogues. The fidelity of fluxes in the XMM-Newton source catalogues could be further improved by using the source spectrum, or hardness ratio, to calculate the ecf.

Acknowledgements. We thank Matteo Guainazzi, Martin Stuhlinger and Simon Rosen for useful comments. We gratefully acknowledge the XMM-Newton Survey Science Centre, a consortium of 10 European institutes, for the production and public provision of the 2XMM catalogue. We would like to acknowledge the contribution of all the calibrators of the EPIC and RGS instruments on XMM-Newton, who have helped to achieve the self-consistency between the instruments that we see today. S.M., A.M.R. and S.S. acknowledge direct support from the UK STFC research council. We thank the referee for providing comments that improved this paper.

\section{References}

den Herder, J. W., Brinkman, A. C., Kahn, S. M., et al. 2001, A\&A, 365, 7 Ehle, M., \& Altieri, B. 2001, SOC note XMM-SOC-CAL-PL-0001 Erd C. 2000, SOC note XMM-SOC-PS-TN-0038

Jansen, F., Lumb, D., Altieri, B., et al. 2001, A\&A, 365, 1

Kendziorra, E., Bihler, E., Grubmiller, W., et al. 1997, SPIE, 3114, 155

Kendziorra, E., Colli, M., Kuster, M., et al. 1999, SPIE, 3765, 204

Kuster, M., Benlloch, S., Kendziorra, E., \& Briel, U. G. 1999, SPIE, 3765, 673

Lumb, D. H., Warwick, R. S., Page, M., \& De Luca, A. 2002, A\&A, 389, 93

Mateos, S., Barcons, X., Carrera, F. J., et al. 2005, A\&A, 444, 79

Mateos, S., Warwick, R. S., Carrera, F. J., et al. 2008, A\&A, 492, 51

Read, A. M., Sembay, S. F., Abbey, T. F., \& Turner, M. J. L. 2006, Proceedings of The X-ray Universe 2005, 26-30 September 2005, El Escorial, Madrid, Spain, ed. A. Wilson (Noordwijk: ESA Publications Division), ESA SP-604, 2,925

Saxton, R. D., Denby, M., Griffiths, R. G., \& Neumann, D. M. 2003, AN, 324, 138

Strüder, L., Briel, U., Dennerl, K., et al. 2001, A\&A, 365, 18

Stuhlinger, M., Kirsch, M. G. F., Santos-Leo, M., et al. 2008, Status of the XMM-Newton instrument cross-calibration with SASv7.1, XMM-SOCCAL-TN-0052

Turner, M. J. L., Abbey, A., Arnaud, M., et al. 2001, A\&A, 365, L27

Watson, M. G., Schröder, A. C., Fyfe, D., \& Page, C. G. 2008, A\&A, 493, 339 